\title{
VALIDACIÓN DE UN INSTRUMENTO PARA MEDIR LA CREATIVIDAD EN ADOLESCENTES SOBRESALIENTES
}

\author{
Arelli García Mendoza, Pedro Antonio Sánchez Escobedo, \\ Angel Alberto Valdés Cuervo ${ }^{1}$
}

\begin{abstract}
RESUMEN
Este reporte documenta el proceso de validación de un instrumento para medir la creatividad de adolescentes yucatecos. Para esto, se utilizó el instrumento Evaluación Multifactorial de la Creatividad (EMUC) (Sánchez, 2006), el cual se administró a 3 grupos de 21 estudiantes cada uno; estos grupos fueron cualitativamente diferentes: alumnos sobresalientes de secundaria, estudiantes de primer semestre de la Facultad de Arquitectura y alumnos regulares de secundaria.
\end{abstract}

Los resultados indicaron que la EMUC es una prueba confiable, con una alfa de Cronbach de .8643 . En cuanto a su validez externa, esta prueba muestra resultados generales diferenciales entre los grupos, y por tanto puede discriminar a las personas creativas de las que no lo son. La creatividad visomotora, se correlacionó positiva y significativamente con la creatividad aplicada en los 3 criterios evaluados: fluidez, flexibilidad y originalidad y se aprecia independencia entre la creatividad aplicada y la creatividad verbal.

\footnotetext{
${ }^{1}$ Universidad Autónoma de Yucatán. Correo electrónico: vcuervo@tunku.uady.mx
} 


\section{INTRODUCCIÓN}

Este trabajo aborda el desarrollo y validación de un instrumento par medir la creatividad en adolescentes sobresalientes. En México no existen instrumentos validos ni confiables que permitan conocer el nivel de creatividad real de los adolescentes sobresalientes. Como respuesta a esta problemática, en esta investigación se pretenden establecer los principales criterios para evaluar la creatividad de una manera multidimensional (verbal, visomotora y aplicada), y no de manera parcial, como es el caso de muchos instrumentos existentes.

Como menciona Sánchez (2006), la identificación, detección y referencia de los niños con habilidades sobresalientes en México es una actividad primitiva, surgida de políticas nacionales a mediados de los 90. Pese al reconocimiento de la importancia que para el desarrollo del país tiene la incorporación de alumnos sobresalientes a los sistemas de educación superior y a la formación de científicos, no existen métodos, instrumentos y procedimientos con bases empíricas comprobadas para la identificación de estos niños.

De acuerdo con Zacatelco (2003), los individuos sobresalientes son aquellos que poseen una superioridad cognoscitiva intelectual, de creatividad y de alta motivación que los hace diferentes de las personas de su mismo grupo y los hace potencialmente viables para contribuir de manera especial con su sociedad. Para Treffinger la capacidad sobresaliente puede ser definida como "un potencial para realizar creativamente, sobre un periodo sostenido de tiempo, en un área que interesa a una persona, la solución a una problemática real” (citado por Castro, Oyanadel, Paez y Quintanilla, 2003, p.13). 
Como se puede apreciar, una idea común en las dos definiciones anteriores es que las personas sobresalientes tienden a ser creativas, por lo que es necesaria la existencia de instrumentos validos y confiables que permitan medir precisamente este constructo.

En la actualidad existen muchas pruebas para medir la creatividad del individuo, siendo las más conocidas el Test de Torrance y la batería de pruebas de Guilford. Con respecto al primer test, Penagos (2000) afirma que coexisten varios problemas que desde una óptica global impiden que lo medido por el test de Torrance sea confiable, por ejemplo: la creatividad es un fenómeno temporal, y no estable; es decir, el ver, plantear y solucionar problemas requiere de diferentes inversiones de tiempo los cuales varían entre las personas y en la persona misma. Otro problema es que la creatividad no sucede de manera aislada sino en un entorno social y en áreas específicas. Por lo tanto, para que un instrumento pueda realmente medir la creatividad debe de considerar las características y el contexto del individuo, lo cual al parecer no se contempla del todo en el test de Torrance.

Por su parte, Chaur (2005) menciona que alrededor de estas dos baterías de pruebas se han desarrollado muchas otras que matizan y subdividen aquellos factores en función de las propias experiencias y visiones, aunque la base sigue siendo las propuestas inicialmente señaladas. Una de las críticas a la batería de Guilford y sus derivados que realiza Chaur (2005), es el hecho de que permite mediciones de los factores de creatividad, pero no posibilita la unificación de estos en un solo índice que permita la comparación objetiva de los resultados.

En este estudio se pretendió determinar las propiedades psicométricas de la EMUC (Evaluación Multifactorial de la Creatividad), calibrar y especificar los criterios de calificación de la EMUC, así como explorar y describir si las dimensiones a evaluar 
son dependientes o independientes (las dimensiones son: creatividad visomotora, aplicada y verbal).

\section{Acotación Del Concepto De Sobresaliente}

Dailey (1984), fue de los primeros en cuestionar, en el habla inglesa, la terminología respecto a las personas con superioridad en algunos o todos los aspectos de la vida. Por ejemplo, el término genio se usaba inicialmente para referirse a quienes eran muy inteligentes, con un coeficiente intelectual (CI) de más de 180 puntos. En el idioma español, López (1994) refiere que con puntajes por encima de 130 se usaba el término sobredotado o brillante.

Las personas sobresalientes han sido descritas de muchas maneras. Witty (citado por Jay y Swerdlik, 2001, p. 282) describió de manera sucinta al individuo sobresaliente como "uno cuyo desempeño es notable de manera consistente en cualquier área valuada en forma positiva". Los criterios para ser considerado sobresaliente han incluido capacidad intelectual, pensamiento creativo, capacidad de liderazgo, capacidad para ejecutar artes y otras aptitudes mecánicas o psicomotoras.

Asimismo, la enciclopedia Gale de Psicología (2001), define a una persona sobresaliente como extremadamente creativa. Para la Organización Mundial de la Salud, es sobresaliente el que tiene un CI mayor de 130 y que además presenta una buena capacidad creativa y motivación intrínseca para el aprendizaje. Cada una de las características que hace de una persona el ser reconocida por tener aptitudes sobresalientes, variará en proporción dependiendo de cada persona, así pues un niño puede tener un CI de 135, una creatividad promedio y una motivación intrínseca para el aprendizaje muy superior al promedio; y otro niño puede presentar un CI de 150, muy 
alta creatividad y una motivación intrínseca para el trabajo superior al promedio y ambos serán niños con aptitudes sobresalientes. Por esto, los sobresalientes forman un grupo que dista mucho de ser homogéneo (citado por Grupo CERPA, 2003).

El estudio más extenso de los sobresalientes fue llevado a cabo en 1921 por Lewis M. Terman en la Universidad de Stanford. Usando la edición de 1916 de la Stanford-Binet, Terman y sus colegas comenzaron el proyecto de investigación longitudinal identificando 1528 niños cuyos CI de 140 o más los colocaban dentro del $1 \%$ superior en el país en funcionamiento intelectual. Terman hizo un seguimiento de estos niños sobresalientes durante el resto de su propia vida, tomando medidas de desarrollo físico y social, rendimiento, rasgos de carácter, libros leídos e intereses reactivos. También incluía entrevistas con los padres, maestros y los propios sujetos. Terman publicó por primera vez algunos de sus hallazgos cuatro años después de que había comenzado el estudio. En general, los sobresalientes tendieron a mantener su capacidad intelectual superior. Además, de tener tasas de mortalidad menores y una salud mental y física mejor que sus contrapartes que no eran sobresalientes. Tendieron a sostener opiniones políticas y sociales moderadas, eran exitosos en sus carreras educativas y vocacionales y cometieron menos crímenes que aquellos que no eran sobresalientes (Jay y Swerdlik, 2001).

\section{Identificación de niños sobresalientes}

Soto (2003), establece que dos de las evaluaciones para la identificación de niños sobresalientes son la de inteligencia y la de creatividad. La primera proviene de la evidencia de un alto rendimiento académico o pruebas de inteligencia, ya que la medición del CI es una de las formas de obtener la capacidad intelectual de un alumno; 
la evaluación de la creatividad se centra en el historial académico del alumno y en sus producciones, debido a la falta de una definición clara del término creatividad.

Por su parte, Blanco (2001), menciona que la identificación de niños sobresalientes es un proceso en el que deben analizarse conjuntamente los resultados de pruebas formales y de observaciones sistemáticas sobre comportamiento y realizaciones de los alumnos. Las pruebas objetivas o formales son las psicométricas, las cuales miden el CI y que deben ser aplicadas siempre por personal especializado; estas pruebas pueden ser: de inteligencia general, de aptitudes específicas, de creatividad y de ejecución o rendimiento. Las más comunes son la de inteligencia general, dentro de los cuales se encuentran The Stanford-Binet Test of Intelligence, The Wechsler Intelligence Scale for Children-Revised (WISC-R) y The Wechsler Preschool and Primary Scale for Intelligence (WPPSI).

Las pruebas de aptitudes específicas, son instrumentos compuestos por una batería de pruebas que miden diferentes capacidades y aptitudes del sujeto. Diversos países han diseñado, validado y estandarizado pruebas de identificación. En México, la prueba SAGES-2 (Screening Assesment for Gifted Elementary and Middle School Student - Second Edition) es una de las pruebas de aptitudes específicas diseñada en Estados Unidos de América que se ha estandarizado y validado en la población mexicana, logrando con ello un fuerte impacto en la Educación Especial y la Psicología (Orta y Sánchez, 2003).

Los test de creatividad tienen gran importancia para la determinación del sobresaliente, aunque no tienen excesiva fiabilidad, ya que como se sabe no están estandarizados ni validados para la población mexicana. El más conocido es el Torrance Test of Creative Thinking, el cual comprende dos partes: una figurativa y otra verbal; y contemplan cuatro factores, la fluidez, la flexibilidad, originalidad y 
elaboración. Sin embargo, una de las principales limitaciones de esta prueba es que fue diseñada para una población limitada, específicamente para los niños de primaria (Duarte, 1997).

\section{Teorías sobre la creatividad}

Según Corbalán, Martínez y Donolo (2003) “existe una confluencia importante entre las teorías actuales de la creatividad que, aún desde paradigmas y métodos diversos, están haciendo propuestas perfectamente compatibles e integradoras” (p.15).

Dentro de las teorías psicológicas de creatividad son significativas: la teoría de la creatividad incremental, la cual considera el arraigo o la experiencia del individuo a su destreza y el desarrollo gradual de su trabajo anterior, por medio de un proceso de pequeños y continuos saltos (Weisberg, 1989). De igual manera, se puede citar la teoría de la transferencia, asociada al desarrollo intelectual creativo, motivado por el impulso intelectual de estudiar y encontrar solución a los problemas por medio de la interacción de las dimensiones del pensamiento compuesto por factores, contenidos y productos mentales que producen la transmisión creativa, es decir, la comunión de las dimensiones mencionadas (Guilford, 1891).

Algunas teorías mencionadas por Solar (1991) son: la teoría humanista, en donde los factores sociales e interpersonales cumplen un papel importante para el desarrollo o el bloqueo a la creatividad. También se menciona la teoría factorial, en donde se ubica a Guilford y Torrance, y en la que se estudia el comportamiento creador por métodos experimentales y teóricos. Más recientemente, se nombra la teoría neuropsicofisiológica, basada en la lateralización y codificación de los hemisferios cerebrales. 
Por su parte, Chacón (2005) afirma que dos de las teorías en donde se han realizado más investigaciones son las de Guilford y la de Torrance. La teoría denominada análisis de factor, intelecto y creatividad se le atribuye a Guilford, en ésta se mencionan tres categorías: contenido, operación y productos. Dentro de la categoría de operación, sobresale el factor general de producción divergente que se considera una operación creativa, que está representado por la fluidez, la flexibilidad y la originalidad de los procesos de pensamiento. La otra teoría de gran influencia es la de la educación y la creatividad, del psicólogo educativo Paul Torrance, quien realizó múltiples investigaciones en relación con el pensamiento divergente y desarrolló diferentes pruebas sobre los procesos del pensamiento creativo, sobre las cualidades de los productos y de las personalidades creativas.

En sí, las diferentes teorías reafirman la creatividad como cualidad del ser humano para construir mundos posibles y como tal, debe estudiarse, fortalecerse y estimularse.

\section{Pruebas de creatividad}

Anastasi (1998) indica que un número creciente de psicólogos y educadores han reconocido al fin que el talento creador no es sinónimo de inteligencia académica y que los tests que proporcionan un CI rara vez lo abarcan. Por su parte, Aiken (2003) menciona que en ocasiones se afirma que la inteligencia por arriba del promedio es necesaria pero no suficiente para la productividad creativa. Más allá de un nivel mínimo de inteligencia, el desempeño creativo parece depender más de la motivación y las habilidades especiales que de la habilidad mental general. Por consiguiente, las investigaciones de la creatividad conducidas durante los pasados 40 años se han 
concentrado en identificar otras características cognoscitivas y afectivas que distinguen a la gente creativa de la no creativa.

En este campo de la creatividad, dos de las baterías de pruebas más destacadas son: los Tests del proyecto de aptitudes de la Universidad de California del Sur de Guilford, y los tests de Torrance de pensamiento creador.

Con relación a los tests del proyecto de aptitudes de la Universidad de California del Sur, Anastasi (1998) alude que a lo largo de dos decenios esta investigación llevó a la formulación del modelo de la estructura del intelecto. Aunque el modelo comprende todas las funciones intelectuales, una importante contribución del proyecto de aptitudes se centró en la selección de producción divergente, sobre la que existía relativamente escasa investigación previa. De hecho el proyecto de aptitudes se inició primariamente como una investigación de razonamiento, creatividad y solución de problemas. En el curso de sus investigaciones de análisis factorial, Guilford elaboró las categorías de pensamiento convergente y divergente. La forma de pensar convergente lleva a una única solución correcta determinada por los hechos dados. Mientras que el pensamiento divergente, por otra parte es algo que se dispara en distintas direcciones.

Las pruebas de Pensamiento Creativo de Torrence consisten en materiales de prueba basados en palabras, al igual que en ilustraciones y en sonidos. Cada subprueba está diseñada para medir algunas o las cuatro características consideradas importantes en el proceso del pensamiento creativo: flexibilidad, originalidad, fluidez y elaboración, y las respuestas son calificadas en tres o más de estas cuatro áreas. La flexibilidad se refiere a la variedad de ideas presentadas y la capacidad para cambiar de un enfoque a otro. La fluidez implica el número de ideas o las respuestas totales que se produjeron en realidad. La elaboración alude a la riqueza del detalle en una explicación verbal o 
representación pictórica. Y la originalidad a la capacidad de producir ideas y figuras que no sean obvias (Jay y Swerdlik, 2001).

A manera de síntesis, Aiken (2003) menciona que las pruebas que han sido diseñadas para evaluar la creatividad con frecuencia tienen correlaciones significativas con las pruebas de CI, y al parecer las primeras no son más efectivas que las últimas para predecir el desempeño creativo. Considerando todas las cosas, una conclusión razonable es que todavía queda por demostrar si es posible construir medidas efectivas de la creatividad.

\section{Indicadores para evaluar la creatividad}

La mayoría de los expertos en el área coinciden en mencionar que los principales indicadores para evaluar la creatividad son: la fluidez, la flexibilidad y la originalidad.

De acuerdo con Guilford (citado por Rodríguez y Romero, 2001) la fluidez, se relaciona con la fertilidad de ideas o respuestas generadas ante una situación. Se refiere al aspecto cuantitativo, en el cual la cualidad no es tan importante en tanto las respuestas sean pertinentes; por su parte, la flexibilidad es la habilidad de adaptar, redefinir, reinterpretar o tomar una nueva táctica para llegar a una solución.

Por último, Guilford (citado por Carevic, 2006) define a la originalidad como la aptitud o disposición para producir de forma poco usual respuestas raras, remotas, ingeniosas o novedosas. 


\section{MÉTODO}

\section{Sujetos}

Debido a la naturaleza del estudio, se determinó utilizar un muestreo por no probabilístico por conveniencia, la muestra estuvo integrada por tres grupos.

El primero conformado por 21 alumnos sobresalientes de segundo grado de secundaria identificados a través del proyecto del CONACYT Fomix YUC-2004-C030013: "Detección y registro de niños de secundaria con capacidades sobresalientes en zonas rurales y suburbanas del estado de Yucatán”, cuyas edades oscilan entre los 12 y 15 años de edad. De estos 21 adolescentes, 5 son de Tizimín, 9 de Valladolid, 1 de Espita, 1 de Río Lagartos, 1 de Peto, 2 de Ticul y 2 de Tzucacab.

El segundo grupo de la muestra estuvo formado por 21 estudiantes de primer semestre de la Licenciatura en Arquitectura que accedieron a participar voluntariamente en el estudio.

Y el tercer grupo estuvo integrado por 21 estudiantes de segundo de secundaria con capacidades escolares promedio los cuales fueron nominados por sus profesores, cuyo criterio de selección fue que tuvieran un promedio general de entre 7 y ocho en sus calificaciones. De los 21 alumnos que conformaron este grupo de la muestra, 10 fueron estudiantes de la escuela Miguel Barrera y 11 de la Técnica \# 4, ambas escuelas están localizadas en el municipio de Tizimín Yucatán.

\section{Instrumento}

El instrumento que se empleó en este estudio fue la Evaluación Multifactorial de la Creatividad (Sánchez, 2006). Este instrumento está dividido en tres apartados, correspondientes a las tres dimensiones de la creatividad que evalúa: la visomotora, la inventiva o aplicada y la verbal. 
En la sección de creatividad visomotora, al adolescente se le presentó una serie de trazos como círculos, curvas y líneas; con los cuales tenía que realizar un dibujo, el tiempo destinado para esta actividad era de tres minutos.

En la sección de creatividad aplicada, al adolescente se le presentaron dos figuras (una cuerda y una sábana), en un máximo de dos minutos, el participante tenía que escribir todos los usos posibles que se le pudiera dar a cada uno de estos artículos.

Finalmente, en el apartado de creatividad verbal, el alumno tenía que inventar y escribir un cuento bien estructurado; es decir que incluyera inicio, desarrollo y final. El cuento debía incluir las seis palabras que se le presentaban al inicio del ejercicio. Para esta actividad se destinó un máximo de cinco minutos.

La codificación de los resultados obtenidos por los sujetos del estudio se analizaron de acuerdo con la siguiente tabla de especificaciones. 
Tabla 1

Tabla de especificaciones de la EMUC

\begin{tabular}{|c|c|c|}
\hline Tipo & Criterio & Evaluación \\
\hline \multirow{9}{*}{$\begin{array}{l}\text { Creatividad } \\
\text { Verbal }\end{array}$} & Fluidez: Número de & $\geq 15$ líneas- 4 puntos \\
\hline & $\overline{\text { líneas utilizadas en el }}$ & 11 - 14 líneas- 3 puntos \\
\hline & cuento. & 7 - 10 líneas- 2 puntos \\
\hline & & $3-6$ líneas- 1 punto \\
\hline & Flexibilidad: Cantidad de & $\geq 6$ ideas -4 puntos \\
\hline & 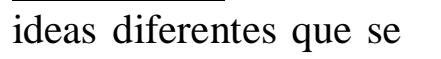 & $4-5$ ideas -3 puntos \\
\hline & generen y adaptación a & 3 - 2 ideas -2 puntos \\
\hline & las existentes. & 1 idea -1 punto \\
\hline & $\begin{array}{l}\text { Originalidad: Fantasía, } \\
\text { situaciones poco } \\
\text { comunes utilizadas en el } \\
\text { cuento. }\end{array}$ & 0- 4 a criterio del lector \\
\hline \multirow{11}{*}{$\begin{array}{l}\text { Creatividad } \\
\text { visomotora }\end{array}$} & Fluidez: Número de & 4 puntos - de 7 trazos en adelante \\
\hline & trazos generados en el & 3 puntos - de 5 a 6 trazos \\
\hline & dibujo. & 2 puntos - de 3 a 4 trazos \\
\hline & & 1 punto - de 1 a 2 trazos \\
\hline & & 0 punto si no emplean ningún trazo \\
\hline & $\begin{array}{l}\text { Flexibilidad: Número de } \\
\text { categorías o }\end{array}$ & $\begin{array}{l}4 \text { puntos - Utilización de } 4 \text { categorías } \\
\text { diferentes en el dibujo }\end{array}$ \\
\hline & $\begin{array}{l}\text { agrupamientos temáticos } \\
\text { diferentes en el dibujo. }\end{array}$ & $\begin{array}{l}3 \text { puntos - Utilización de } 3 \text { categorías } \\
\text { en el dibujo }\end{array}$ \\
\hline & & $\begin{array}{l}2 \text { puntos - Utilización de } 2 \text { categorías } \\
\text { en el dibujo. }\end{array}$ \\
\hline & & $\begin{array}{l}1 \text { punto - Utilización de } 1 \text { categoría } \\
\text { en el dibujo. }\end{array}$ \\
\hline & & 0 puntos - Utilización de 0 categorías \\
\hline & $\begin{array}{l}\text { Originalidad: Grado en } \\
\text { que es novedoso el } \\
\text { dibujo creado. }\end{array}$ & $0-4$ a criterio del lector \\
\hline \multirow{10}{*}{$\begin{array}{l}\text { Creatividad } \\
\text { aplicada }\end{array}$} & Fluidez: Cantidad de & $\geq 10$ usos 4 puntos \\
\hline & usos que le de a cada & $8-9$ usos \\
\hline & objeto. & $5-7$ usos 2 puntos \\
\hline & & $3-4$ usos 1 puntos \\
\hline & & $0-2$ usos 0 puntos \\
\hline & $\begin{array}{l}\text { Flexibilidad: número de } \\
\text { categorías o }\end{array}$ & $\begin{array}{l}4 \text { puntos - Utilización de } 5 \text { categorías } \\
\text { diferentes }\end{array}$ \\
\hline & $\begin{array}{l}\text { agrupamientos temáticos } \\
\text { diferentes. }\end{array}$ & $\begin{array}{l}3 \text { puntos - Utilización de } 3 \text {-4 } \\
\text { categorías diferentes. }\end{array}$ \\
\hline & & $\begin{array}{l}2 \text { puntos - Utilización de } 2 \text { categorías } \\
\text { diferentes }\end{array}$ \\
\hline & & $\begin{array}{l}1 \text { punto - Utilización de } 1 \text { categorías } \\
\text { diferentes. }\end{array}$ \\
\hline & & 0 puntos - Utilización de 0 categorías \\
\hline
\end{tabular}




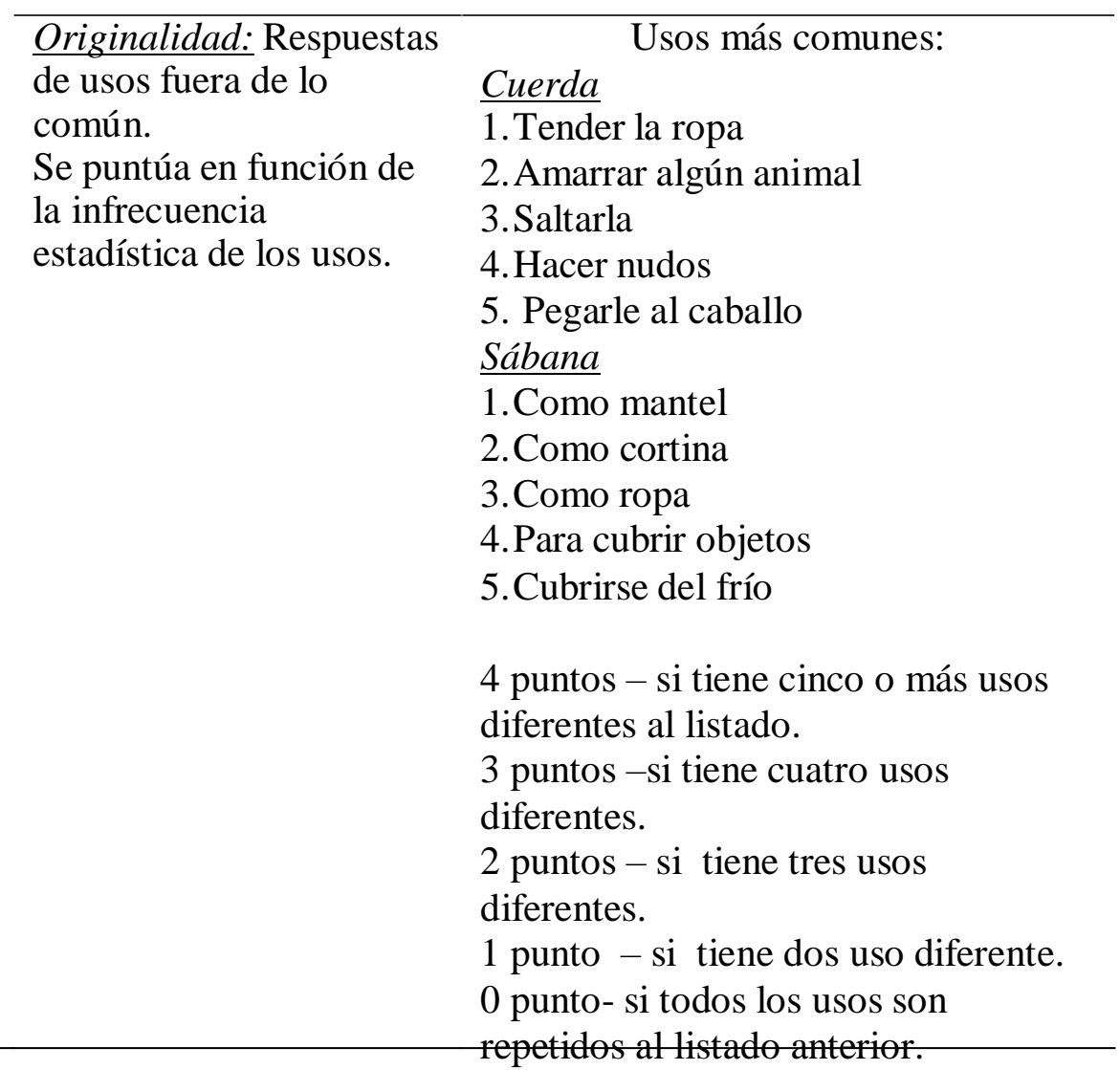

\section{Procedimiento}

El procedimiento realizado fue el siguiente:

Etapa uno: elaboración de una tabla de especificaciones para calificar la prueba.

Tomando en consideración que el instrumento ya estaba diseñado por Sánchez (2006), se decidió elaborar una tabla de especificaciones en la que se detallaron los criterios para calificar cada una de las dimensiones que comprende la prueba. Los criterios fueron elaborados tomando como base las aportaciones teóricas realizadas por los autores Franco (2005) y Granados (2002), asimismo, los criterios de calificación, fueron sometidos a una revisión inter-juez, protagonizada por el personal que intervino en el diseño de la prueba, incluyendo al Dr. Pedro Antonio Sánchez Escobedo. 
Etapa dos: contacto con los tres grupos que conformaron la muestra

Enseguida se efectuaron los trámites y permisos correspondientes para contactar a los tres grupos concernientes de la muestra: los 21 estudiantes de secundaria con capacidades sobresalientes de las zonas rurales y suburbanas del estado de Yucatán (grupo uno); los 21 alumnos del primer semestre de la Licenciatura en Arquitectura (grupo dos) y los 21 alumnos con capacidades académicas promedio de las escuelas Miguel Barrera y la Técnica \# 4, localizadas en el municipio de Tizimín Yucatán (grupo tres).

Etapa tres: administración del instrumento

Se administró la EMUC en las fechas y hora acordadas, a los 21 alumnos sobresalientes ya identificados, a los 21 estudiantes de la Facultad de Arquitectura y a los 21 alumnos de segundo de secundaria con capacidades escolares promedio. En los tres casos se respetaron los tiempos establecidos para contestar cada una de las secciones de la prueba, esto con la finalidad de que los datos obtenidos fueran confiables.

Etapa cuatro: captura de datos

Los datos recabados de los tres grupos que conformaron la muestra, fueron capturados y almacenados en una base de datos. 


\section{RESULTADOS}

Para fines prácticos, al grupo integrado por los estudiantes de primer semestre de la Facultad de Arquitectura, se le ha denominado grupo experto; a los alumnos sobresalientes de segundo de secundaria, grupo sobresaliente; y a los alumnos de segundo de secundaria con capacidades escolares promedio, grupo promedio.

\section{Puntajes de las tres dimensiones}

La tabla 2, muestra las puntuaciones promedio y la desviación estándar de los tres grupos incluidos en el estudio, comparando las medias obtenidas en la dimensión visomotora a través del ANOVA de una vía. Cabe señalar que las puntuaciones de esta dimensión, se obtuvieron sumando los respectivos puntajes de los criterios de fluidez, flexibilidad y originalidad.

Tabla 2

Puntajes índice en la creatividad visomotora $(N=63)$

\begin{tabular}{lcccc} 
Grupo & $n$ & $\bar{X}$ & $F$ & $<p$ \\
& & $(D E)$ & & \\
\hline $\begin{array}{l}\text { Grupo } \\
\text { experto }\end{array}$ & 21 & 9.66 & 19.06 & .001 \\
& & $(1.39)$ & &
\end{tabular}

\begin{tabular}{lcc}
$\begin{array}{l}\text { Grupo } \\
\text { sobresaliente }\end{array}$ & 21 & 9.42 \\
& & $(1.32)$ \\
& & \\
$\begin{array}{l}\text { Grupo } \\
\text { promedio }\end{array}$ & 21 & 6.85 \\
\hline
\end{tabular}

Como se puede ver en el análisis de varianza, existe diferencia significativa entre los tres grupos con relación a la dimensión visomotora $(\mathrm{F}=19.06, \mathrm{p}<.001)$. 
En la tabla 3, se exponen los resultados obtenidos de la comparación múltiple realizada a los tres grupos que conformaron la muestra, tomando como punto de comparación el puntaje obtenido en la dimensión visomotora.

Tabla 3

Comparación múltiple de los grupos en la dimensión visomotora, Post Hoc (LSD)

\begin{tabular}{lcc} 
Grupo & Grupo & Grupo \\
\hline & sobresaliente & promedio \\
Grupo & .23 & $2.80^{*}$ \\
\hline experto & & \\
Grupo & & $2.57^{*}$ \\
\hline
\end{tabular}

sobresaliente

* Los puntajes marcados con asterisco indican diferencias significativas entre los grupos.

Al efectuar el proceso de comparación múltiple LSD se determinó que el grupo experto, integrado por los estudiantes de Arquitectura; y el grupo sobresaliente, formado por los alumnos sobresalientes, obtuvieron las mayores puntuaciones en esta dimensión. Es decir, en el caso de la dimensión visomotora, la prueba no detecta con justicia la alta creatividad en adultos, pero sí es capaz de diferenciar entre pares, o alumnos de la misma edad, ya que como se observa los alumnos sobresalientes tuvieron puntajes significativamente más altos que el grupo representado por los alumnos con capacidades académicas promedio.

En la tabla 4, se detallan las puntuaciones promedio y la desviación estándar de los tres grupos, comparando las medias obtenidas en la dimensión aplicada o inventiva a través del ANOVA de una vía. Cabe señalar que las puntuaciones de esta dimensión, se obtuvieron sumando los respectivos puntajes de los criterios de fluidez, flexibilidad y originalidad. 
Tabla 4

Puntajes índice de la creatividad aplicada $(N=63)$

\begin{tabular}{lcccc}
\cline { 3 - 4 } Grupo & $n$ & $\bar{X}$ & $F$ & $<p$ \\
& & $(D E)$ & & \\
\hline $\begin{array}{l}\text { Grupo } \\
\text { experto }\end{array}$ & 21 & 9.52 & 82.55 & .001 \\
& & $(1.39)$ & & \\
$\begin{array}{l}\text { Grupo } \\
\text { sobresaliente }\end{array}$ & 21 & 6.97 & & \\
& & $(1.31)$ & & \\
$\begin{array}{l}\text { Grupo } \\
\text { promedio }\end{array}$ & 21 & 4.47 & & \\
\hline
\end{tabular}

En el análisis de varianza se muestra que existe diferencia significativa entre los tres grupos con relación a la dimensión de creatividad aplicada o inventiva $(\mathrm{F}=82.55$, $\mathrm{p}<.001)$

En la tabla 5, se exponen los resultados obtenidos de la comparación múltiple realizada a los tres grupos que conformaron la muestra, tomando como patrón de comparación el puntaje obtenido en la dimensión aplicada o inventiva.

Tabla 5

Comparación múltiple de los grupos en la dimensión aplicada, Post Hoc (LSD)

\begin{tabular}{lcc}
\hline Grupo & $\begin{array}{c}\text { Grupo } \\
\text { sobresaliente }\end{array}$ & $\begin{array}{c}\text { Grupo } \\
\text { promedio }\end{array}$ \\
\hline Grupo & $2.54^{*}$ & $5.04^{*}$ \\
experto & & \\
\hline Grupo & & $2.50^{*}$ \\
sobresaliente & & \\
\hline
\end{tabular}

\footnotetext{
* Los puntajes marcados con asterisco indican diferencias significativas entre los grupos.
} 
En este caso, el análisis de comparación múltiple señala que, los sobresalientes tuvieron mayores puntajes que los alumnos con capacidades académicas promedio y los estudiantes de Arquitectura tuvieron mayores puntajes en comparación de los otros dos grupos. Lo que indica que en la dimensión de creatividad aplicada el instrumento realmente ayudó a establecer diferencias significativas entre los tres grupos en cuestión.

La tabla 6, muestra las puntuaciones promedio y la desviación estándar de los tres grupos incluidos en el estudio, comparando las medias obtenidas en la dimensión verbal a través del ANOVA. Cabe señalar que las puntuaciones de esta dimensión, se obtuvieron sumando los puntajes respectivos de los criterios de fluidez, flexibilidad y originalidad.

Tabla 6

Puntajes índice de la creatividad verbal $(N=63)$

\begin{tabular}{lcccc}
\cline { 3 - 4 } Grupo & $n$ & $\bar{X}$ & $F$ & $<p$ \\
& & $(D E)$ & & \\
\hline $\begin{array}{l}\text { Grupo } \\
\text { experto }\end{array}$ & 21 & 9.80 & 33.20 & .001 \\
& & $(1.60)$ & & \\
$\begin{array}{l}\text { Grupo } \\
\text { sobresaliente }\end{array}$ & 21 & 7.38 & & \\
& & $(1.93)$ & & \\
Grupo & & & \\
promedio & 21 & 5.04 & \\
\hline
\end{tabular}

Como se puede ver en el análisis de varianza, existe diferencia significativa entre los tres grupos con relación a la dimensión verbal $(\mathrm{F}=33.20, \mathrm{p}<.001)$.

En la tabla 7, se exponen los resultados obtenidos de la comparación múltiple realizada a los tres grupos que conformaron la muestra, tomando como patrón de comparación el puntaje obtenido en la dimensión verbal. 


\section{Tabla 7}

Comparación múltiple de los grupos en la dimensión verbal, Post Hoc (LSD)

\begin{tabular}{lcc} 
Grupo & Grupo & Grupo \\
\hline & sobresaliente & promedio \\
Grupo & $2.42^{*}$ & $4.76^{*}$ \\
\hline experto & & \\
Grupo & & $2.33^{*}$ \\
\hline
\end{tabular}

sobresaliente

* Los puntajes marcados con asterisco indican diferencias significativas entre los grupos.

Nuevamente, en la dimensión de creatividad verbal, los estudiantes de Arquitectura (grupo experto) obtuvieron mayor puntuación, los alumnos sobresalientes se ubicaron en segundo sitio, y finalmente, los alumnos que integran el grupo promedio, obtuvieron los menores puntajes. Por lo que se puede decir que la EMUC también sirvió para señalar las diferencias existentes entre los tres grupos con relación a esta dimensión.

\section{Confiabilidad}

Para calcular la confiabilidad del instrumento se empleó el alfa de Cronbach. La confiabilidad de la EMUC fue de .86 lo que indica que es un instrumento con alto grado de confiabilidad.

\section{Correlación entre las dimensiones}

Debido a que resultaba importante para la investigación determinar si las dimensiones de la creatividad resultaban a la luz de los resultados empíricos, independientes cada una o bien las tres explicaban un solo y único concepto, se exploraron los resultados a través de una matriz de correlación. 
En la tabla 8, se ilustra con una matriz de correlación, utilizando el estadístico r de Pearson, las relaciones entre las propiedades de cada una de las dimensiones de la prueba.

Tabla 8

Matriz de correlación entre las dimensiones de la EMUC

\begin{tabular}{lccc}
\hline & Visomotora & Aplicada & Verbal \\
\hline Visomotora & - & $.598^{*}$ & $.725^{*}$ \\
\hline Aplicada & & - & $.683^{*}$ \\
\hline Verbal & & - \\
\hline
\end{tabular}

Como puede observarse, todas las dimensiones tienen independencia positivamente baja entre ellas, por lo que se asume que están correlacionadas significativamente, por lo tanto las tres dimensiones se influyen mutuamente entre sí en la misma dirección.

\section{DISCUSIÓN, CONCLUSIONES Y RECOMENDACIONES}

\section{Discusión}

Con relación a los puntajes obtenidos por los miembros de los tres grupos que conformaron la muestra, en las dimensiones: aplicada o inventiva y en la verbal, los estudiantes de Arquitectura tuvieron puntajes significativamente mayores en comparación de los otros dos grupos. Estos resultados son sustentados por la teoría de la creatividad incremental citada por Weisberg (1989) y Penagos (2000), quienes mencionan que la creatividad es una característica del desarrollo, por lo que al crecer se presenta una confrontación que promueve la originalidad y el desarrollo de la creatividad por medio de pequeños y continuos saltos. 
En la sección de creatividad visomotora, la prueba no detecta con justicia la alta creatividad en adultos, esto se debe a que la prueba no es apropiada para adolescentes en etapas tardías o en adultos (Duarte, 1997). Sin embargo, la prueba sí es capaz de diferenciar entre pares o alumnos de la misma edad, ya que como se observa los alumnos sobresalientes tuvieron puntajes significativamente más altos que el grupo representado por los alumnos con capacidades académicas promedio.

De igual forma, los resultados mostraron que los alumnos sobresalientes anteriormente detectados, cuyo CI estaba por encima de 120 puntos, obtuvieron en todas las dimensiones puntajes significativamente mayores a los representados por los alumnos con capacidades académicas promedio, por lo que, en comparación con ese grupo, los estudiantes sobresalientes fueron más creativos, lo cual coincide con lo expuesto por Aiken (2003) quien menciona que con frecuencia las pruebas de CI tienen correlaciones significativas con las pruebas que han sido diseñadas para evaluar la creatividad. De igual manera, estos resultados apoyan la teoría de la transferencia de Guilford (1891), la cual asocia el desarrollo intelectual con el potencial creativo, pues argumenta que existe comunión entre estas dimensiones, ya que la persona motivada por el impulso intelectual de estudiar y encontrar solución a los problemas por medio de la interacción de las dimensiones del pensamiento compuesto por factores, contenidos y productos mentales; produce finalmente la transmisión de creatividad.

Asimismo, el hecho de que los alumnos sobresalientes hayan tenido puntuaciones mayores en las tres dimensiones de la creatividad que evalúa la prueba, en comparación con los alumnos con capacidades académicas promedio, da pie a que se corrobore lo expuesto por Zacatelco (2003), quien señala que los individuos sobresalientes son aquéllos que poseen una superioridad cognoscitiva intelectual y de creatividad que los hace diferentes de las personas de su mismo grupo. 
Los resultados apoyan la utilización de estas tres dimensiones para evaluar la creatividad, ya que aunque los puntajes en las mismas poseen correlación significativa, lo cual indica que evalúan un mismo constructo. Sin embargo, dicha correlación, deja un margen para evidenciar cierta independencia entre los puntajes lo que señala que las tres dimensiones miden aspectos diferentes del constructo creatividad. Lo anterior es una cualidad deseable de los instrumento de medición (Hogan, 2003).

Finalmente, se hace hincapié en la necesidad de continuar la investigación al respecto de las estrategias y técnicas disponibles para identificar a los estudiantes con alto potencial creativo en la escuela.

\section{Conclusiones}

Con base en los resultados, se puede concluir que:

1. La creatividad debe de evaluarse de una manera multidimensional, y no únicamente de manera parcial, como la mayoría de las pruebas existentes que tan sólo contemplan la dimensión visomotora. Pues los resultados arrojaron que las dimensiones visomotora, aplicada o inventiva y la verbal, se correlacionan significativamente y de manera lineal, por lo que se puede decir que son dimensiones dependientes, es decir en la medida que una de ellas aumenta, las otras también lo hacen; pero no por el hecho de ser variables dependientes, dejan de tener relativa independencia entre sí, por lo cual es necesario el estudio de estas tres dimensiones de la creatividad por separado.

2. Con relación a la validez de criterio de la prueba, se puede decir que en efecto, la EMUC sirvió para discriminar a las personas creativas de las que no lo eran, pues en ninguna de las dimensiones (visomotora, aplicada y verbal), el grupo tres integrado por estudiantes con capacidades académicas promedio, obtuvo puntuaciones que superaran a cualquiera de los otros dos grupos de la muestra. Sin embargo, es necesario señalar 
que La Evaluación Multifactorial de la Creatividad no detecta con justicia la alta creatividad visomotora en adultos, pero sí es capaz de diferenciar entre pares, o adolescentes de la misma edad.

3. El alfa de Cronbach obtenido fue de .86, por lo que la EMUC tiene un nivel aceptable de confiabilidad.

4. Los puntajes obtenidos por los alumnos sobresalientes muestran que, en efecto, el alto potencial creativo tiene relación con el puntaje de CI, y ambas son características esenciales para que una persona pueda ser catalogada como sobresaliente.

5. Con base en las conclusiones anteriormente planteadas, se puede decir que la Evaluación Multifactorial de la Creatividad es un instrumento válido y confiable, por lo que se recomienda su empleo de manera informada y responsable a los profesionales que así lo decidan.

\section{Recomendaciones}

Una vez expuestos los resultados y las conclusiones del estudio, se presentan algunas recomendaciones que se espera sean de utilidad para las personas involucradas directamente en el proyecto, así como para cualquier persona que decida realizar investigaciones relacionados directamente con este constructo.

1. Se recomienda que se sigan realizando investigaciones que aborden el tema de la medición de la creatividad, pues como se ha observado, medir este constructo se torna difícil ya que la creatividad implica necesariamente salirse de la norma o de lo cotidiano, es por eso que se exhorta a que se sigan realizando investigaciones, pues de esta manera se ayudará a sustentar lo aquí escrito, o bien a refutar los resultados presentados.

2. Se recomienda que en próximos estudios se conciba a la creatividad como una cualidad humana multidimensional y por tanto sean tomadas en cuenta las dimensiones 
verbal, visomotora y aplicada, pues de esta manera se obtendría un panorama más general de este constructo y no se caería en encasillar a la creatividad en alguna de estas tres dimensiones.

3. Debido a que el instrumento no detecta con justicia la alta creatividad visomotora en adultos, pero sí es capaz de diferenciar entre pares, o alumnos de la misma edad, se recomienda que en estudios posteriores se reformule la sección de la prueba relacionada con la creatividad visomotora de tal manera que, al igual que en las otras dimensiones, se puedan establecer claramente y de manera significativa las diferencias existentes entre diferentes grupos de población.

4. Asimismo, se insta a que futuros estudios den pie a que se analice a mayor profundidad las correlaciones existentes entre las tres dimensiones creativas. 


\section{REFERENCIAS}

Aiken, L. (2003). Test psicológicos y evaluación. México: Pearson

Anastasi, A. (1998). Test psicológicos. México: Aguilar

Blanco, M. (2001). Guía para la identificación y seguimiento de alumnos Superdotados. España: Cisspraxis.

Brown, F. (2000). Principios de la medición en psicología y educación. México: Manual Moderno.

Carevic, M. (2006). Creatividad. [En red] Recuperado el 13 de noviembre de 2006, en: http://www.psicologia-online.com/articulos/2006/creatividad.shtml

Castro, P., Oyanadel, C., Paez, A. Y Quintanilla, R. (s/f). Implicancias de una Educación especial para superdotados. Recuperado el 27 de mayo de 2003, de http://www.geocities.com/Athens/Thebes/1663/pablo1.htm.

Corbalán, J., Martínez, F., y Donolo, D. (2003). Manual Test CREA. Inteligencia creativa. Una medida cognitiva de la creatividad. España: TEA Ediciones.

Chacón, Y. (2005). Una revisión crítica del concepto de creatividad. Revista Electrónica Actualidades Investigativas en Educación. [En red]. Recuperado de http://revista.inie.ucr.ac.cr/articulos/1-2005/articulos/creatividad.pdf.

Chaur, J. (2005). Diseño conceptual de productos asistido por ordenador: Un estudio analítico sobre aplicaciones y definición de la estructura básica de un nuevo programa. Tesis para obtener el grado de doctor, Universidad Politécnica de Catalunya, Barcelona, España.

Dailey, R., Hallahan, D. y Kauffman, J.(1984). Special education for today .EUA: Prentice-Hall.

Duarte, E. (1997). Niveles diferenciales de creatividad gráfica y su relación con el tipo de personalidad en estudiantes universitarios. Tesis para obtener el grado de 
Maestría en Educación Superior, Universidad Autónoma de Yucatán, Mérida, Yucatán, México.

Franco, C. (2005). Relación entre las variables autoconcepto y creatividad en una muestra de alumnos de educación infantil. [En red] Recuperado de http://redie.uabc.mx/vol8no1/contenido-franco.html

Gale Encyclopedia of Psychology. (2001). Giftednes.(2a. ed.).Gale Group. Recuperado el 20 de mayo de 2003, de http://www.findarticles.com/cf_dls/g2699/0004/2699000483/pl/article.jht

Gardner, H. (1993). Mentes Creativas. España: Paidós.

Granados, M. (2002). Definición empírica de los factores de fluidez ideativa, originalidad y creatividad: relaciones con la personalidad. Tesis de doctorado. España: Universidad Complutense de Madrid.

Grupo CERPA. (s/f). Adaptación curricular individual para alumnos con altas Capacidades. Recuperado el 27 de mayo de 2003, de http://www.grupocerpa.com/gcpages/gcacialtascapas.htm

Guilford, J. (1981). Tres aspectos del intelecto. EUA: Irvington Publishers

Hernández, Fernández y Baptista (2003). Metodología de la investigación. México: Mc Graw Hill.

Hogan, T. (2003). Pruebas psicológicas. Una introducción práctica. México: Manual Moderno.

Jay y Swerdlik. (2001). Pruebas y evaluación psicológicas. México: Mc Graw Hill. López, M. (1994). Estudio, mito y realidad del niño sobredorado. México: Lupus Inquisidor.

Mitjáns, A. (1993): ¿Cómo evaluar la creatividad? Revista Cubana de Psicología. 
Vol.10. No 2-3. Ciudad de La Habana

Montes, Amavizca y Penagos (2000). Test de habilidades creativas. Revista psicología [en red]. Recuperado de http://homepage.mac.com/penagoscorzo/creatividad_2000/creatividad7.ht

Orta, Y. y Sánchez, P. (2003). Habilidades verbales de niños con habilidades sobresalientes: un estudio exploratorio preliminar en Yucatán. Artículo no publicado, Universidad. Autónoma de Ciudad Juárez y Universidad Autónoma de Yucatán. México.

Ortiz, A. (1997). Indicadores para la educación y el desarrollo de la creatividad profesional. Recuperado el 28 de febrero de 2007, de http://www.monografias.com/trabajos13/indicrea/indicrea.shtml.

Parnes, S. (1973). Guía del comportamiento creador. Módulo 1. México: Editorial Diana.

Penagos, J. (2000) Creatividad, una aproximación. Revista Psicológica. [En red] Recuperado el 18 de septiembre de 2006, de http://homepage.mac.com/penagoscorzo/creatividad_200/creatividad1.htm Rodríguez, M y Romero, J. (2001). La creatividad en collage: su validación social. [En red] Recuperado el 13 de noviembre de 2006, en: http://www.psicologia-online.com/ciopa2001/actividades/60/

Salkind, N. J. (1998). Métodos de investigación. México: Prentice Hall

Sánchez, P. (2006). Detección y registro de niños de secundaria con capacidades sobresalientes en zonas rurales y suburbanas del estado de Yucatán. Reporte final. Consejo Nacional de Ciencia y Tecnología, Fondos Mixtos 
YUC-2004-C03-0013.

Santibáñez, R. (2001). Manual para la evaluación del aprendizaje estudiantil. México: Trillas.

Solar, M. (1991). Creatividad: Desafío a la función docente universitaria. España: Paidea.

Soto, T. (2003). Sobredotación: contextualización y experiencias pedagógicas en España. Psicología de educación para padres y profesionales. [En red] Recuperado el 12 de septiembre de 2006, en: http://www.psicopedagogia.com/articulos/?articulo=444

Taylor, C. (1959). The nature of the creative process.. EUA: Hastings House Weisberg, (1989). Creatividad, genio y otros mitos. España: Ediciones Labor. Woodman, R \& Scoenfeld, L. (1990). An interactionist model of creative behavior. Journal of creative behavior, 24(4), 279-289

Zacatelco, F. (2005). Modelo para la identificación de niños sobresaliente en las escuelas de educación primaria. Tesis de doctorado. México: Universidad Nacional Autónoma de México. 
Apéndice

\section{EMUC: Evaluación Multifactorial de la Creatividad Instrucciones}

A continuación se presentan una serie de ejercicios que evaluaran tu capacidad creativa en tres dimensiones:

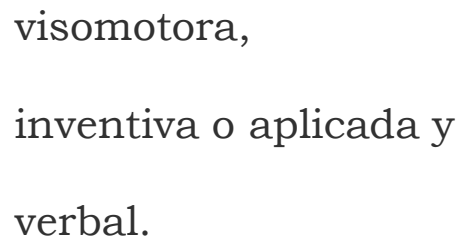

Sigue las instrucciones del aplicador ya que cada actividad tiene un tiempo límite predeterminado.

¡Haz tu mejor esfuerzo!

\section{Datos sociode mográficos del estudiante}

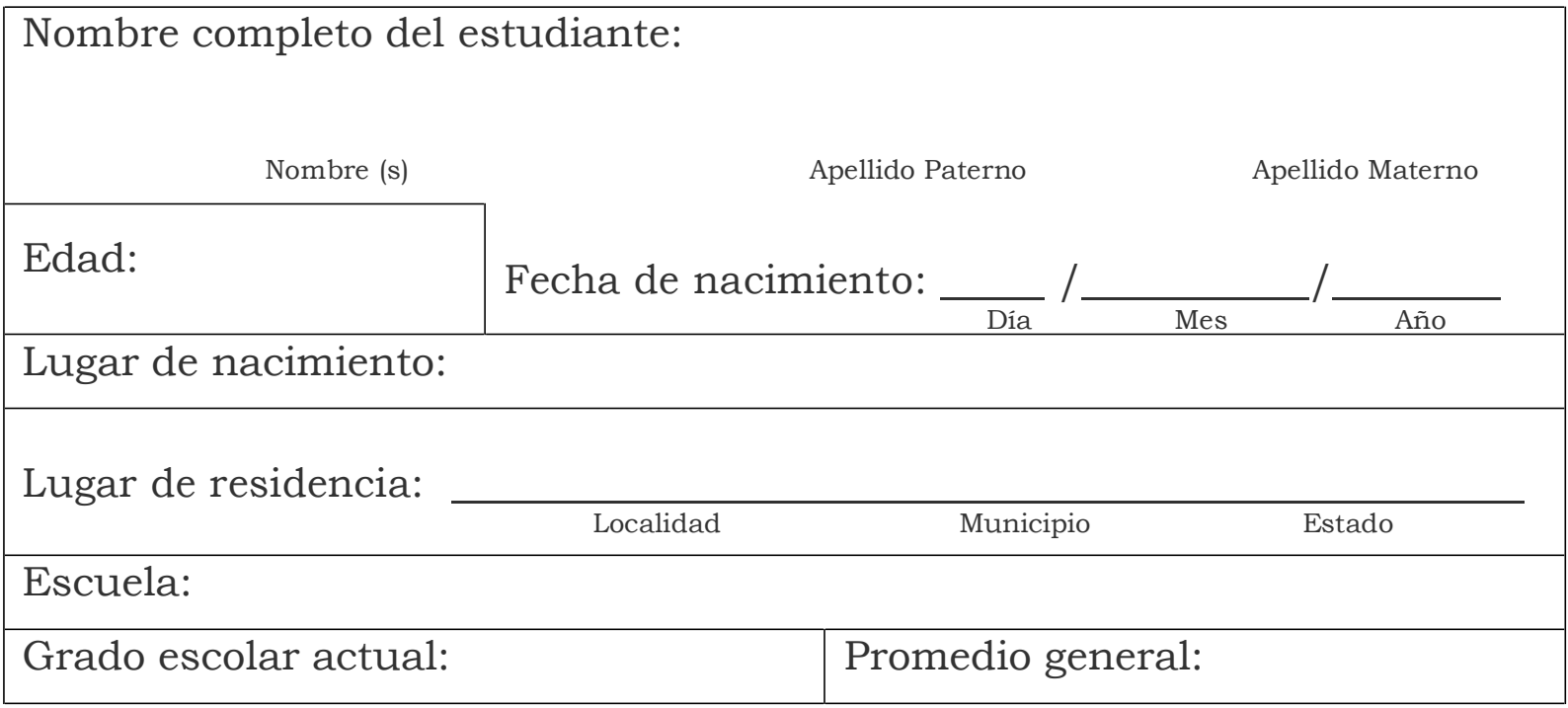




\section{Creatividad visomotora}

Instrucciones
Crea un dibujo en el recuadro de la izquierda, utilizando todos los trazos que se
encuentran en el cuadro de la derecha; puedes agregar más formas. Tienes
TRES minutos para hacer el dibujo.

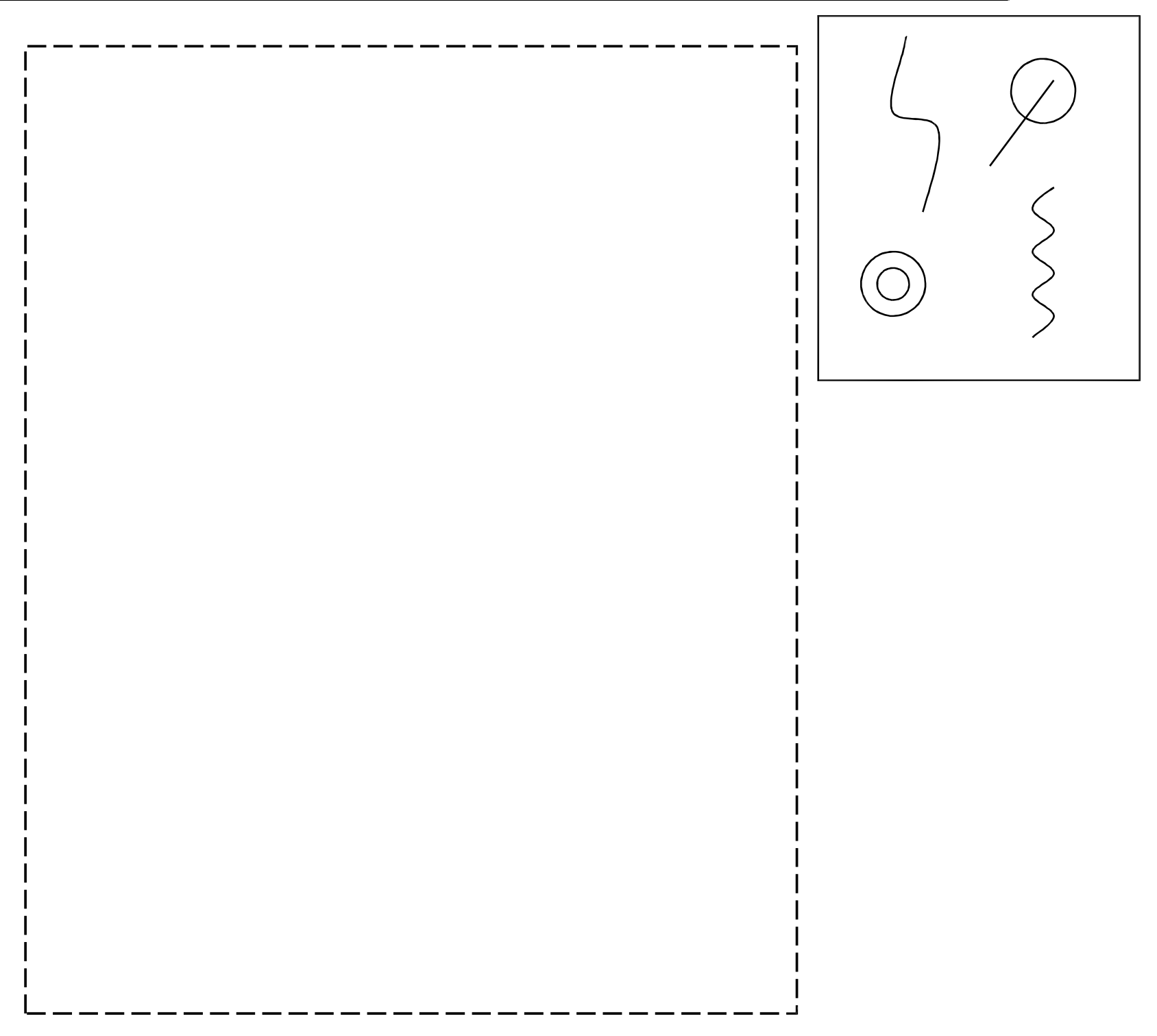




\section{Creatividad aplicada (1)}

Instrucciones
A continuación se te presenta una figura. Piensa y escribe todos los usos
posibles que le puedas dar a este objeto. Escribe todos los usos que se te
ocurran. Tienes DOS minutos para completar esta tarea.

Cuerda

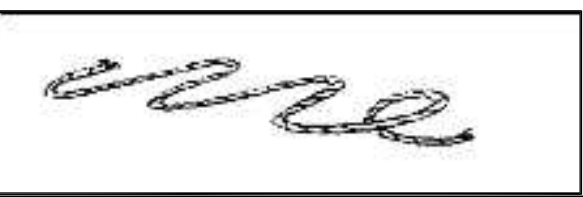

\section{Creatividad aplicada (2)}

\section{Instrucciones}

A continuación se te presenta una figura. Piensa y escribe todos los usos posibles que le puedas dar a este objeto. Escribe todos los usos que se te ocurran. Tienes DOS minutos para completar esta tarea.

Sábana

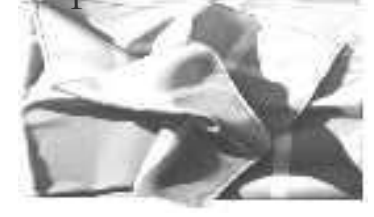

\begin{tabular}{|l|}
\hline \\
\hline \\
\hline \\
\hline \\
\hline \\
\hline
\end{tabular}




\section{Creatividad Verbal}

\section{Instrucciones}

A continuación se presentan 6 palabras. Con ellas inventa un cuento que incluya todas las palabras. Debes escribir un inicio, el desarrollo de la historia y un final. Tienes CINCO minutos para terminar la tarea.

Playa, computadora, oso, cepillo, otoño y bata.

\begin{tabular}{|l|}
\hline \\
\hline \\
\hline \\
\hline \\
\hline \\
\hline \\
\hline \\
\hline \\
\hline
\end{tabular}




\section{(ब) (1)}

Este texto está protegido por una licencia Creative Commons $\underline{4.0}$.

Usted es libre para Compartir — copiar y redistribuir el material en cualquier medio o formato- y Adaptar el documen- to remezclar, transformar y crear a partir del material- para cualquier propósito, incluso comercialmente, siempre que cumpla la condición de:

Atribución: Usted debe reconocer el crédito de una obra de manera adecuada, proporcionar un enlace a la licencia, e in- dicar si se han realizado cambios. Puede hacerlo en cualquier forma razonable, pero no de forma tal que sugiera que tie- ne el apoyo del licenciante o lo recibe por el uso que hace. 